\title{
FENOLOGÍA DE Caesalpinia pulcherrima (L.) Sw. EN UN JARDÍN BOTÁNICO URBANO DE LIMA, PERÚ
}

\section{PHENOLOGY OF Caesalpinia pulcherrima (L.) Sw. IN AN URBAN BOTANICAL GARDEN OF LIMA, PERU}

\author{
Rosario Castro-Muñoz ${ }^{1}$, Viviana Castro-Cepero² y Aldo Ceroni-Stuva ${ }^{3}$
}

\begin{abstract}
Resumen
Se estudió la fenología de Caesalpinia pulcherrima L., arbusto o árbol pequeño muy apreciado tanto ornamentalmente por la belleza de sus flores, como medicinalmente por sus propiedades curativas, desde Enero 2006 hasta Diciembre 2007, determinándose tres estadios de floración: "botón floral”, “floración total” y “flor marchita”, que mostraron un patrón anual de floración entre los meses de Febrero y Julio, continuando con estallidos esporádicos de aparición de flores entre Setiembre y Octubre. Los estadios “fruto apareciendo" y "fruto verde", presentaron un patrón anual de fructificación entre los meses de Febrero y Mayo, para el primer estadio y entre Febrero y Setiembre para el segundo. El estadio "fruto maduro" tuvo un patrón continuo de fructificación, presentándose con mayor intensidad entre los meses de Julio y Setiembre. En relación a la fenofase vegetativa, el estadio “más del 50\% de hojas nuevas”, se presentó durante casi todo el año con una mayor intensidad en los meses de Febrero y Marzo, mientras que el estadio “más del 50\% de hojas viejas” se presentó de manera constante durante casi todo el año sin mostrar ningún pico regular de aparición en los dos años estudiados. El estadio "pocas hojas" sólo se presentó en el último año entre los meses de Setiembre y Noviembre, sin mostrar un patrón regular en los dos años. El análisis multivariado de correspondencia canónica, mostró que la temperatura media tiene una influencia positiva sobre la fenofase de floración, mientras que la humedad relativa media mostró tener influencia en la aparición de los estadios "fruto maduro" y “más del 50\% de hojas viejas".
\end{abstract}

Palabras clave: Fenología, fenofases, estadio fenológico, jardín botánico.

\begin{abstract}
Caesalpinia pulcherrima (L.) phenology was studied between January 2006 and December 2007. This plant is a shrub or little tree much appreciated both ornamentally for the beauty of its flowers and medicinally for its healing properties. In this study it was determined that the three stages of bloom: "flower bud", "total bloom" and "wilted flower" showed an annual flowering pattern from February to July, followed by sporadic outbreaks of bloom from September to October. The "appearing fruit" and "green fruit" stages presented an annual pattern of fruiting between February and May for the first stage and between February and September for the second. The "ripe fruit" stage had a continuous fruiting pattern, with higher intensity between July and September. With regard to the vegetative phenophase: the "Over 50\% of New Leaves" stage appeared almost the entire year, with higher intensity in the months of February and March, while the "More than $50 \%$ of Older Leaves" stage also appeared almost the whole year, without showing regular peaks in the two years. The "Few Leaves" stage only appeared in the last year between September and November. Multivariate canonical correspondence analysis showed that the average temperature had a positive influence on phenophase flowering and that the average relative humidity had influence on the occurrence of the following stages: "Ripe Fruit" and "More than $50 \%$ of Older Leaves".
\end{abstract}

Key words: Phenology, phenophases, phenological stage, botanical garden.

\section{Introducción.}

La fenología es una disciplina que trata del tiempo de aparición de fenómenos periódicos característicos en el ciclo vital de los organismos (ej. En las plantas, los estadios de floración), y su relación con los factores ambientales (SECF, 2005). Las observaciones de las fases fenológicas han sido reconocidas como bio-indicadores al cambio climático global y como 
una de las formas más simples de monitorear las respuestas ecológicas al cambio de clima. (Alvarado et al., 2002; Rosenzweig et al., 2008). Para muchas especies, los periodos de floración y defoliación están correlacionados a la temperatura (Harper et al., 2004). Otras especies tienen periodos de floración influenciados por la longitud del día o la precipitación (Primack \& Miller-Rushing, 2009). Los jardines botánicos localizados en grandes áreas urbanas presentan temperaturas más elevadas que los que están en las áreas circundantes, por el efecto llamado "isla del calor urbano” (NERAG, 2001). Este incremento del calor en los jardines botánicos en ciudades grandes, proveen percepciones útiles en los cambios ecológicos que podrían ocurrir en muchas áreas rurales como resultado de un calentamiento global. En áreas urbanas existe más contaminación y más altas concentraciones de varios gases que en las áreas rurales, el valor predictivo de los jardines de la ciudad en comparación al campo podría verse afectado (Gregg et al., 2003). Sin embargo, los efectos de la contaminación sobre la fenología de las plantas parecen ser menores a los efectos de temperatura, luz, y precipitación (Cleland et al., 2006).

El Jardín Botánico “Octavio Velarde Núñez” de la Universidad Nacional Agraria - La Molina (UNALM) inicia sus actividades a partir de 1933, actualmente realiza investigación científica en taxonomía, conservación ex situ de recursos vegetales y ecología; realizando estudios fenológicos de diversas especies y entre ellas se encuentra Caesalpinia pulcherrima (L.) Sw., conocida comúnmente como "ponciana enana", "ave roja del paraíso", "flamboyán enano" (Soto, 2010), “angel sisa” (Mejía \& Rengifo, 1995). Esta especie pertenece a la familia Fabaceae, arbusto, de copa irregular y dispersa, rala, extendida, con ramas delgadas en forma de paraguas. Su fuste presenta ejes múltiples, este y las ramas poseen espinas y muchas lenticelas, tanto en el tronco como en ramas. La corteza es delgada, de color blancuzca a gris claro. Esta planta mide entre 3.7-4.3 m de altura; las hojas son abruptamente bipinnadas, alternas, de 3 a 9 pares de pinnas por hoja y de 6 a 12 hojuelas por pinna; las pinnas son redondeadas en el ápice, pálidas en el envés; las flores son rojas o amarillas; sus frutos son vainas dehiscentes al madurar. Sus flores son muy vistosas, se presentan en inflorescencias terminales, de gran colorido variando desde amarillo hasta rojo, inclusive con tonos mixtos. Es una planta andromonoica, produce frutos secos dehiscentes, tipo vaina, color café cuando se encuentran maduros, son coriáceos, aplanados, de aproximadamente $10 \mathrm{~cm}$ de largo, en forma de espiral al abrirse. Las semillas presentan una forma aplanada, de aproximadamente $0,8 \mathrm{~cm}$ de largo; de testa color verde oscuro, lisa, brillante, lustrosa y muy dura (Rojas \&Torres, 2009).

C. pulcherrima es muy apreciada por la belleza de sus flores (pulcherrima significa “la más bella”), siendo muy utilizada en las ciudades como cerco-vivo en la arborización de las calles, parques y jardines (Lorenzi \& Souza, 2008). Esta especie no solo es importante desde el punto de vista ornamental, sino también, como medicinal por sus diversas propiedades curativas, tales como el tratamiento de la bronquitis y el asma (Pullaiah, 2006), disminución de la fiebre y desinflamación del hígado (Mejía \& Rengifo, 1995). Sus extractos tienen actividad antimicrobiana (Sudhakar et al., 2006), actividad repelente contra Aedes aegypti, mosquito que causa la malaria (Govindarajan et al., 2011) y actividad anti ulcérica (Takawale et al., 2011). De sus frutos se extraen taninos que se utilizan como tinte (Paguagua \& Soto, 2010).

C. pulcherrima está ampliamente distribuida tanto en regiones tropicales como sub-tropicales en América, África y Asia. En América se encuentra desde México hasta Sudamérica y también en las Antillas. Su origen no está aún establecido, probablemente tiene su origen en el norte de Centroamérica y se encuentra naturalizada en los trópicos del Viejo Mundo. En Nicaragua es común en bosques secos, a veces se observa en pastizales y a orillas de caminos en todas las zonas del país, 0-650 msnm, ocasionalmente se siembra a mayor altura (Paguagua \& Soto, 2010). En México es común en bosques tropicales caducifolios (Zepeda \& Velazquez, 1999). Este arbusto crece bien en todo tipo de suelos incluyendo el arenoso, arcilloso, limoso, suelos ácidos o alcalinos. Es altamente tolerante a la sequía, moderadamente tolerante a las sales, pero es intolerante a la inundación. A pesar que puede crecer en lugares sombreados, requiere de sol directo para florear. Se propaga por semillas, es un arbusto siempre verde (Selvam, 2007).

Los estudios fenológicos son fundamentales para el conocimiento del ciclo anual de las especies, indicar la época de producción de ciertas semillas, así como para identificar las influencias ecológicas sobre los diferentes estadios de la planta. A pesar de su importancia, los estudios fenológicos de esta especie aún no se han desarrollado en el hemisferio sur. Este trabajo tiene por objetivo estudiar el comportamiento fenológico de Caesalpinia pulcherrima (L). Sw. en el Jardín Botánico de la UNALM ubicado en un área urbana de Lima, contribuyendo a validar los patrones de florecimiento, fructificación y foliación, así como relacionar los estadios fenológicos con la temperatura media y humedad relativa media durante el tiempo de estudio.

\section{Materiales y métodos.}

Ubicación.

Este trabajo de investigación se realizó en el Jardín Botánico “Octavio Velarde Núñez” ubicado en la Universidad Nacional Agraria La Molina (UNALM) distrito de La Molina, localizado en la zona sureste de 
la ciudad de Lima, Perú. Geográficamente está a $12^{\circ} 05^{\prime} 06^{\prime \prime}$ S. y 7657’00” O y a $238 \mathrm{msnm}$. El clima localizado en la ciudad de Lima, a una altitud de 243.7 msnm, corresponde de acuerdo al sistema de Holdridge elaborado en 1960, a la zona de vida denominada “desierto desecado subtropical” (dd-S) (Holdridge, 2000). El área del Jardín Botánico es de aproximadamente de 2 ha y cuenta con especies endémicas e introducidas. El clima presenta temperatura anual promedio de $20^{\circ} \mathrm{C}$ (temperatura mínima mensual promedio de $16^{\circ} \mathrm{C}$ y máxima de $22^{\circ} \mathrm{C}$ ), humedad relativa (H.R.) promedio de $81 \%$ (H.R. máxima promedio $94.8 \%$, H.R: mínima promedio $71.20 \%$ ), total anual de 1707 horas de sol y precipitación total anual de $12 \mathrm{~mm}$ (según datos del Observatorio Meteorológico Alexander Von Humboldt 472AC278 (UNALM).

Metodología.

Se seleccionaron 8 plantas de esta especie y se realizaron visitas mensuales durante 2 años, desde enero del 2006 hasta diciembre 2007. Pinheiro \& Almeida (2000) enfatizan que la colecta de datos de un año debe ser evitada porque un año puede ser atípico, pudiendo no retratar las fenofases típicas de la especie. Se evaluaron los estadios fenológicos presentes en las diferentes épocas del año siguiendo la escala de Campbell (1970) citado por Rondón (1994) (Tabla 1). Los datos reproductivos fueron registrados cuantitativamente indicando el número de plantas por cada estadio, y también el porcentaje de cada estadio fenológico por planta. Los datos vegetativos se registraron indicando el número de plantas en cada estadio fenológico. Se construyeron fenogramas para ilustrar los estadios fenológicos en el tiempo.

Correlación de los estadios fenológicos con los factores ambientales.

Los datos meteorológicos fueron proporcionados por el Observatorio Meteorológico "Alexander Von Humboldt" de la Facultad de Ciencias de la Universidad Nacional Agraria La Molina. Se establecieron dos tipos de correlaciones: 1) correlaciones lineales para todos los estadios fenológicos reproductivos en base al porcentaje de

Tabla 1. Fenofases y Estadios Fenológicos evaluados en este estudio

\begin{tabular}{ll}
\hline FENOFASE & ESTADIO FENOLOGICO \\
\hline 1.Floración & Botón Floral a \\
2.Floración & Floración Total b \\
3.Floración & Flor Marchita c \\
4.Fructificación & Fruto Apareciendo d \\
5.Fructificación & Fruto Verde e \\
6.Fructificación & Fruto Maduro f \\
7.Foliación & Pocas Hojas \\
8.Foliación & Más del 50\% de Hojas Nuevas \\
9.Foliación & Más del 50\% de Hojas Viejas
\end{tabular}

Leyenda: a: yema floral, capullo; b: flor totalmente abierta; c: flor sin turgencia, seca; d: ovario desarrollado con flor marchita presente; e: vainas inmaduras de color verde; $\mathrm{f}$ : vainas de color marrón. estadio fenológico por copa con los factores de temperatura y humedad para el mes de ocurrencia (T0), y para los siete meses anteriores a la ocurrencia T-1 (mes anterior), T-2 (dos meses anteriores) , T-3 (tres meses anteriores), T-4 (cuatro meses anteriores), T-5 (cinco meses anteriores), T-6 (seis meses anteriores) y $\mathrm{T}-7$ (siete meses anteriores). Las correlaciones fueron establecidas siguiendo el coeficiente de correlación de Pearson (calculados por el programa Excel); y 2) también se estableció la correlación que hay entre los estadios fenológicos Reproductivos y Vegetativos basadas en los porcentajes de individuos y la temperatura media y la humedad relativa media del aire del mismo mes; los datos fueron analizados mediante la prueba de ordenación multivariada denominada Correspondencia Canónica. Antes de aplicar esta prueba, se realizó la trasformación de los datos fenológicos aplicando la siguiente fórmula: $\operatorname{Ln}(\mathrm{x}+1)$. Para el análisis de datos se utilizó el programa para análisis de datos científicos PAST versión 1.84. No se consideró precipitación porque en esta localidad la precipitación anual apenas alcanzó los $24.5 \mathrm{~mm}$, además las plantas recibieron riego periódico, que podría alterar los análisis.

\section{Resultados.}

En el Jardín botánico, los meses registrados con las temperaturas más frías se encuentran entre junio y setiembre y los meses registrados con las temperaturas más cálidas se encuentran entre Enero y Abril (Figura 2).

El comportamiento fenológico reproductivo de $C$. pulcherrima durante los años 2006 y 2007 fue similar entre los dos años: el coeficiente de correlación (r) entre los estados fenológicos en ambos años fue de 0.74 y entre los factores climáticos en ambos años fue de 0.99. El mayor porcentaje de individuos en los estadios de Botón Floral, Floración Total y Flor Marchita ocurrió en mes de abril para ambos años (Figura 1), con el 100\% de la población manifestando estos tres estadios floración al mismo tiempo. En

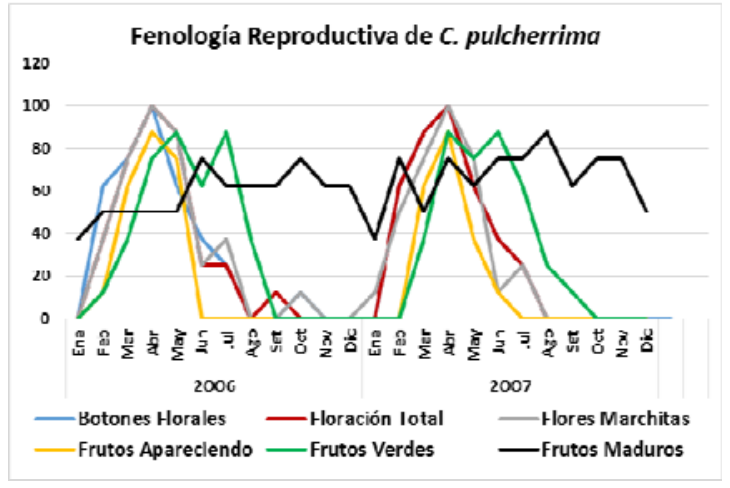

Figura 1. Fenograma reproductivo de $C$. pulcherrima en base al porcentaje de plantas por cada estadio fenológico. 


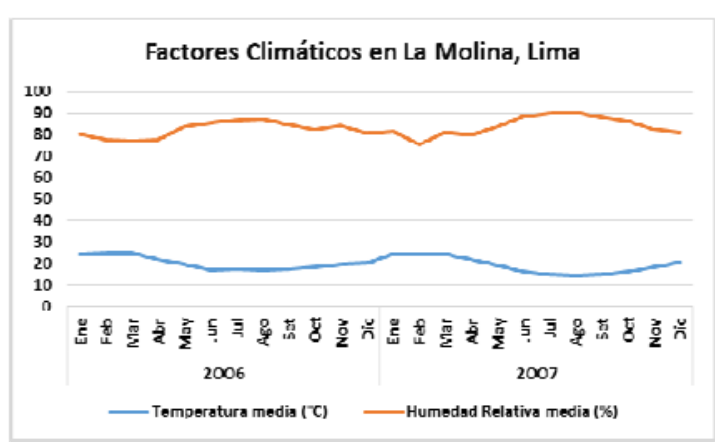

Figura 2. Registros de Temperatura media $\left({ }^{\circ} \mathrm{C}\right)$ y Humedad Relativa media (\%). Registrados por el Observatorio Alexander Von Humboldt de la Universidad Nacional Agraria - La Molina, Lima - Perú.

promedio tuvieron una proporción de $17 \%$ de Botones Florales, 10\% de Floración Total y $18 \%$ de Flores Marchitas por copa de planta (Figura 3). Los datos meteorológicos (figura 2) indican que la máxima temperatura media $\left(24.7^{\circ} \mathrm{C}\right)$ y la mínima humedad relativa media (77\%) se registraron en marzo., es decir un mes antes a la máxima expresión de toda la fenofase de floración. En La Molina, en ambos años en promedio, el porcentaje de plantas que estuvo en los estadios de Botón Floral, Floración Total y Flor Marchita a lo largo del año fue de $31.2 \%$, el 31.2\% y el $30.2 \%$ respectivamente, por lo que ningún estadio de floración muestra ser superior a otro. El estadio Botón Floral en ambos años, se registró desde Febrero hasta Julio, y entre los meses Agosto y Enero la observación de este estadio fue escasa $(12.5 \%$ en Octubre) o nula. El estadio Floración Total en ambos años se presentó de manera similar al de Botón Floral, desde Febrero hasta Julio, y observándose en el mes de Setiembre en el $12.5 \%$ de la copa, y en los demás meses fue nula. El estadio de Flor Marchita también se registró de Febrero a Julio, y también la floración escasa (12.5\% en Octubre) o nula entre los meses de Agosto hasta Enero. En relación a la fenofase de fructificación (Figura 1): el mayor porcentaje de individuos con Fruto Apareciendo ocurrió en el mes de Abril, con el $87.5 \%$ de los individuos manifestando este estadio fenológico, y con el $24 \%$ de la copa de la planta en promedio expresando este estadio (figura 3). De la misma manera que en el caso de la fenofase de floración, la máxima expresión del estadio Fruto Apareciendo ocurre en un mes donde se registra la máxima temperatura y la mínima humedad relativa para ambos años. En la Figura 1 se aprecia que el estadio Fruto Apareciendo se produce desde Febrero hasta Mayo, siendo Junio el mes en el que la temperatura media desciende por debajo de los $18^{\circ} \mathrm{C}$ y la humedad relativa media asciende por encima del 85\%. En relación al estadio de Fruto Verde, la formación de este estadio se dio entre los meses de
Abril y Mayo, obteniéndose el mayor porcentaje de individuos que manifestó este estadio en los meses de Abril y Mayo, con un $87.5 \%$ de los individuos manifestando este estadio fenológico. En relación al porcentaje de frutos verdes por copa de la planta, el mayor porcentaje se dio en el mes Junio con un 31.2\% en el año 2006 y un $29.7 \%$ en el año 2007. En ambos años, el estadio de Fruto Verde ocurrió entre los meses de Febrero y Agosto (Figura 3) y en este último mes se registraron en ambos años los valores más bajos de temperatura media $\left(17.3^{\circ} \mathrm{C}\right.$ en el 2006 y $14.2^{\circ} \mathrm{C}$ en el 2007) y los valores más altos de humedad relativa media (87\% en el 2006 y 90\% en el 2007) respectivamente. La cantidad de frutos verdes disminuye notablemente a partir de agosto y desde setiembre hasta enero no se observaron frutos verdes, reiniciando su aparición hasta febrero. Los frutos maduros se observaron durante todo el periodo de estudio, los mayores porcentajes de individuos con frutos maduros se dio en los meses de Junio (75\%) y Octubre (75\%) en el año 2006 y en el mes de agosto del 2007 (87.5\%). El mayor porcentaje de Frutos maduros por copa de planta se produjo entre los meses de Julio y Setiembre, obteniéndose porcentajes de hasta $48.7 \%$. El comportamiento de la fenofase vegetativa durante los dos años de observación, se muestra en la Figura 4. El mayor porcentaje de individuos en el estadio Más del 50\% de hojas nuevas ocurrió en los meses de febrero y marzo, en el que el $100 \%$ de los individuos mostró este estadio en ambos años de estudio, meses que coinciden con las más altas temperaturas. Este estadio se presenta durante casi todo el año, excepto entre los meses de octubre y noviembre. El estadio Pocas Hojas no se observó en el 2006, mientras que en el 2007 se observó que el 100 $\%$ de las plantas se encontraban en este estadio entre los meses de Octubre y Noviembre, disminuyendo su porcentaje en Diciembre (37.5\%). El estadio Más del $50 \%$ de hojas viejas, también se presentó durante casi todo el año, alcanzando su pico máximo en Setiembre

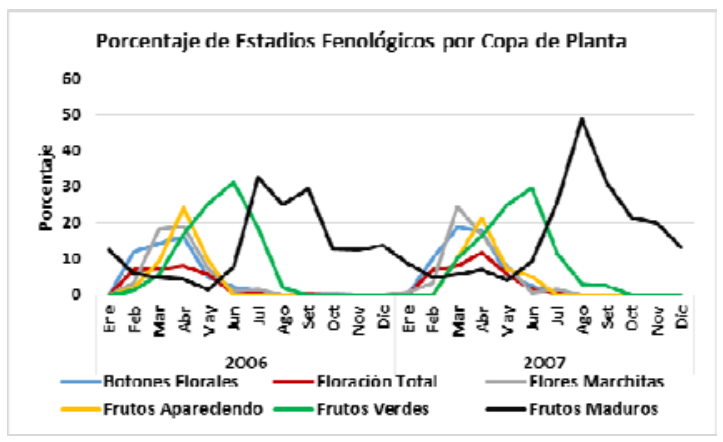

Figura 3. Fenograma reproductivo en base al porcentaje de los estadios fenológicos de Floración y Fructificación por copa de cada planta. 


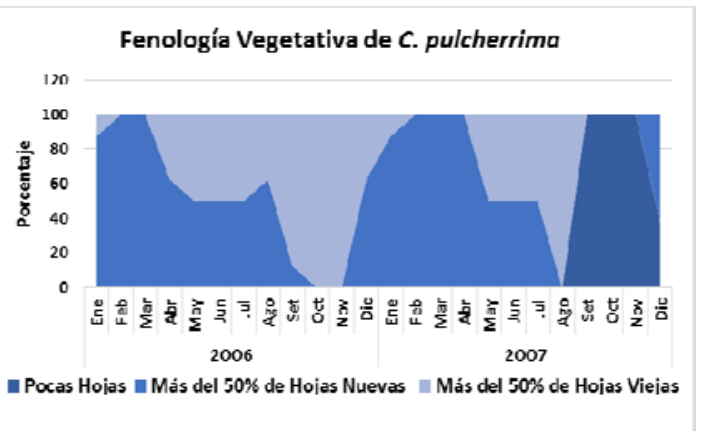

Figura 4. Fenofase Vegetativa de Caesalpinia pulcherrima L. en La Molina.

y Octubre del 2006, en las que $100 \%$ de las plantas se encontraron en este estadio; todo lo contrario sucedió en el 2007, en los que en estos mismos meses no se presentó, siendo reemplazado por el estadio Pocas Hojas, meses en los que la temperatura media no supera los $20^{\circ} \mathrm{C}$.

Correlaciones.

1) Evolución de la Correlación.

Al analizar la evolución de las correlaciones entre los porcentajes de cada uno de los estadios fenológicos por copa de planta y la temperatura (Figura 5), se nota que los mejores coeficientes se alcanzaron cuando los datos fenológicos son correlacionados con los de temperatura: del mes anterior ( $\mathrm{T}-1)$ para los estadios de Botón Floral ( $\mathrm{r}=$ 0.88) y Floración Total $(r=0.88)$; de dos meses anteriores para los estadios de Flor Marchita $(r=0.78)$ y Fruto Apareciendo $(r=0.75)$; de tres a cuatro meses anteriores para el estadio Fruto Verde $(r=0.80+2)$ y, de 6 meses anteriores para el estadio de Fruto Maduro $(r=0.80)$. Todos los estadios de la fenofase de floración y los estadios de Fruto Apareciendo y Fruto Verde muestran un patrón estacional con distintos grados de demora de respuesta en función a la

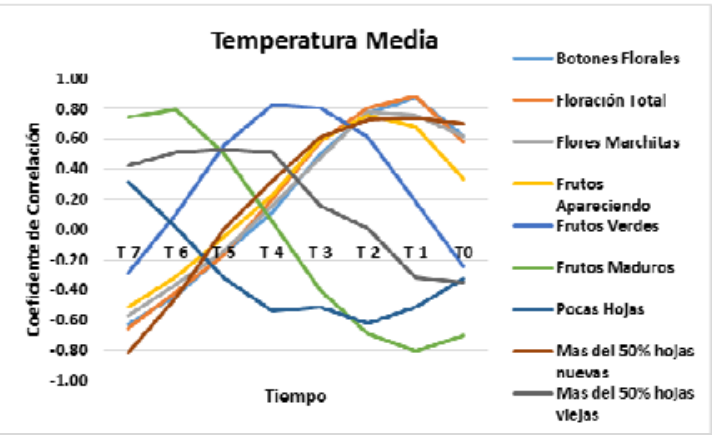

Figura 5. Evolución de las correlaciones entre los estadios fenológicos y la temperatura media del aire, en el mismo mes (T0), en el mes anterior ( $\mathrm{T}$ 1 ), dos meses antes (T-2), tres meses antes (T-3), cuatro meses antes (T-4), cinco meses antes ( $\mathrm{T}-5)$, seis meses antes (T-6) y siete meses antes (T-7). temperatura.

2) Análisis de Correspondencia Canónica entre los Factores Ambientales (Temperatura media ${ }^{\circ} \mathrm{C}$ y Humedad Relativa media H.R. \%).

Los estadios fenológicos de las fenofases reproductivas (Figura 7) y vegetativa (Figura 8) fueron correlacionados con los factores climáticos de temperatura y humedad relativa mediante el análisis de correspondencia canónica. Para los estadios fenológicos de Botón Floral, Floración Total, Flor Marchita, y Fruto Apareciendo, la temperatura es el parámetro de significancia. En relación a la fenofase vegetativa (Figura 8), se observa que el estadio Pocas Hojas, no muestra relación con la temperatura, y muy baja relación con la humedad. Más de 50\% de hojas nuevas muestra una alta correlación con la temperatura registrada tanto en el mismo mes de estudio como en el mes anterior. El estadio Más de $50 \%$ de hojas viejas, tiene mayor correlación con la humedad relativa que con la temperatura.

\section{Discusión.}

Patrones Fenológicos.

Los resultados de este estudio revelan que C. pulcherrima, tiene un patrón de floración anual entre los meses de Febrero y Agosto manifestando pequeños estallidos de floración entre los meses de Setiembre y Octubre; según Newstrom et al., (1994), las especies que tienen patrones de floración anual tienen un principal episodio de floración por año, que pueden incluir dos variaciones: 1) un patrón anual pulsado, con pausas embebidas dentro del principal periodo de floración y 2) con adicionales estallidos tardíos o precoces de baja amplitud que esporádicamente ocurren fuera del principal periodo de floración. Esta última variación fue registrada en el presente estudio para C. pulcherrima y también fue registrada para C. echinata en Brasil (Borges et al., 2009). En los estudios realizados en latitudes del hemisferio norte, revelan que la floración de $C$. pulcherrima ocurrió entre Julio y Abril en el Valle Central de Costa Rica (Rojas \& Torres, 2009), y entre Agosto y Octubre en el bosque deciduo tropical en Jalisco, México (Bullock \& Solis-Magallanes, 1990), en ambos casos se reveló también un patrón de floración anual; sin embargo sus periodos de floración son distintos a los hallados en este estudio, por tener condiciones climáticas distintas. Lamentablemente no se han encontrado trabajos de fenología de $C$. pulcherrima en latitudes cercanas a $12^{\circ} \mathrm{S}$ que nos sirva como patrón de comparación.

El patrón de fructificación resultó difícil de establecer porque tanto el estadio de Fruto apareciendo, como el estadio Fruto Verde mostraron un patrón de fructificación anual, entre los meses de Febrero y Agosto, mientras que el estadio Fruto Maduro mostró un patrón de fructificación continuo, este último patrón es similar al registrado en San 


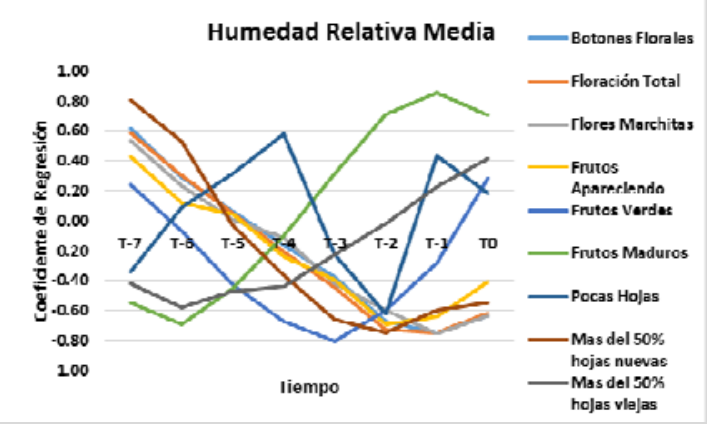

Figura 6. Evolución de las correlaciones entre los estadios fenológicos y la Humedad Relativa media del aire, en el mismo mes (T0), en el mes anterior (T-1), dos meses antes (T-2), tres meses antes (T-3), cuatro meses antes (T-4), cinco meses antes (T-5), seis meses antes (T-6) y siete meses antes (T-7) .

Antonio de los Baños, La Habana, Cuba (Fuentes et al., 2000). En este estudio el periodo de fructificación resultó ser largo, lo que concuerda con lo mencionado por Jordano (1993) que dijo que en comunidades tropicales, las especies individuales tienden a tener periodos de fructificación largos, con un promedio mayor de cuatro meses. A nivel de comunidad, esta especie da frutos durante todo el año, también fructifica en épocas en que muchas otras especies no lo hacen. Fenner (1998) menciona que aquellas

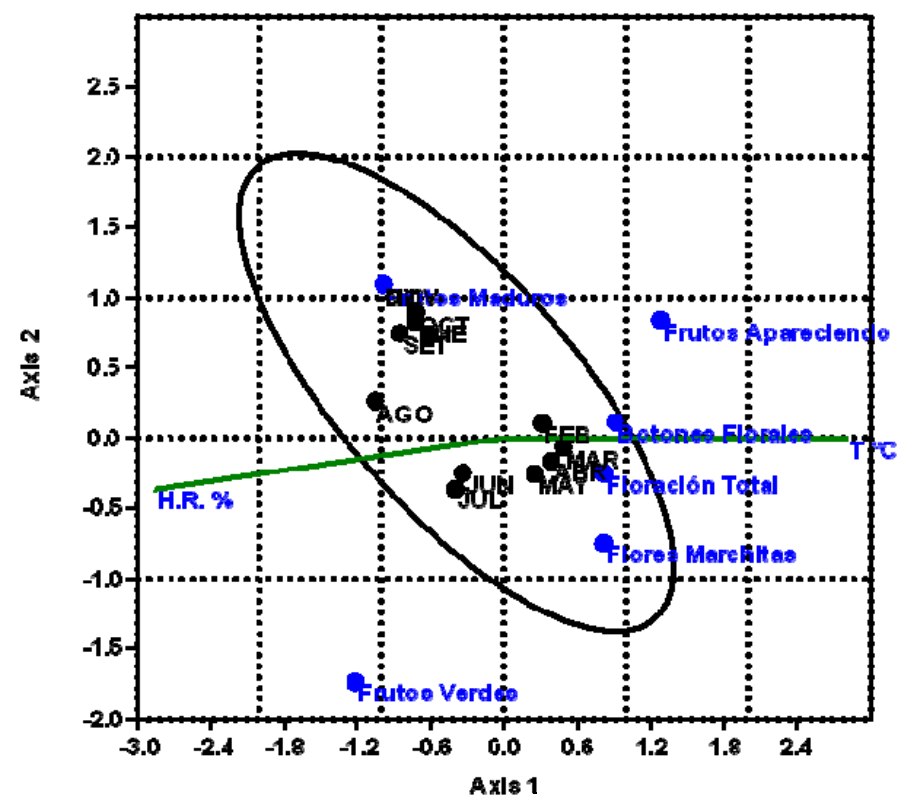

Figura 7. Análisis de Correlación Canónica de la Fenología Reproductiva de Caesalpinia pulcherrrima L. y los parámetros ambientales $\left(\mathrm{T}{ }^{\circ} \mathrm{C}\right.$ y H.R. entre los meses de Enero 2006 y Diciembre 2007. especies que fructifican en estaciones de escasez general tienen un rol central en el mantenimiento del funcionamiento de la comunidad y la diversidad; y su remoción podría causar empobrecimiento de la comunidad. Por lo anteriormente mencionado, esta especie parece ser muy importante en el mantenimiento del ecosistema, sin embargo se necesitarían hacer estudios a nivel de comunidad para confirmarlo.

En relación al porcentaje de individuos que producen frutos maduros, este se presentó de manera muy irregular durante los años de estudio, sin embargo si se analiza el porcentaje de frutos maduros por planta, el mayor porcentaje se registró entre los meses de Julio y Setiembre, coincidiendo con los meses de más baja temperatura y elevada humedad, mientras que en los demás meses su producción fue baja.

En relación a la fenología vegetativa, no se puede generalizar si la foliación tiene un patrón continuo o estacional, porque en el primer año de estudio (2006) la foliación fue continua, pero en el segundo año, hubo un periodo de defoliación mostrando pocas hojas durante tres meses (de Set hasta Nov), la caída de hojas normalmente está ligada con algún cambio en las condiciones ambientales, tales como disponibilidad de agua (Williams et al., 1997), temperatura (Rusch, 1993) o fotoperiodo (Loubry, 1994), como no se tiene certeza de la causa se recomendaría realizar estudios fenológicos de varios años. Lo que si se aprecia en este estudio es que la mayor parte del año se presentó foliación tanto de hojas nuevas como de viejas. Usualmente la foliación está ligada con algún factor climático tal como la lluvia (Bullock \& SolisMagallanes, 1990), la irradiación (Wright \& Van Schalk, 1994), la temperatura (Brooke et al., 1996) o el fotoperiodo (Loubry, 1994), cuando el agua no es el factor limitante los otros factores pueden tener un rol importante. En este trabajo el agua no fue un factor limitante, porque se regaba dentro del jardín, sin embargo las altas temperaturas en el año (febrero y marzo) están ligadas a los picos más altos de producción de hojas nuevas. En $C$. pulcherrima el patrón de desarrollo de hojas está influenciado por la temperatura, se registró un aumento de la producción de nuevas hojas durante los períodos de temperaturas más altas.

Correlaciones entre Fases Fenológicas y Datos Meteorológicos.

Las correlaciones lineales entre el porcentaje de cada estadio reproductivo por copa con la temperatura indican que entre el factor temperatura y el inicio de floración hay un mes de demora de 


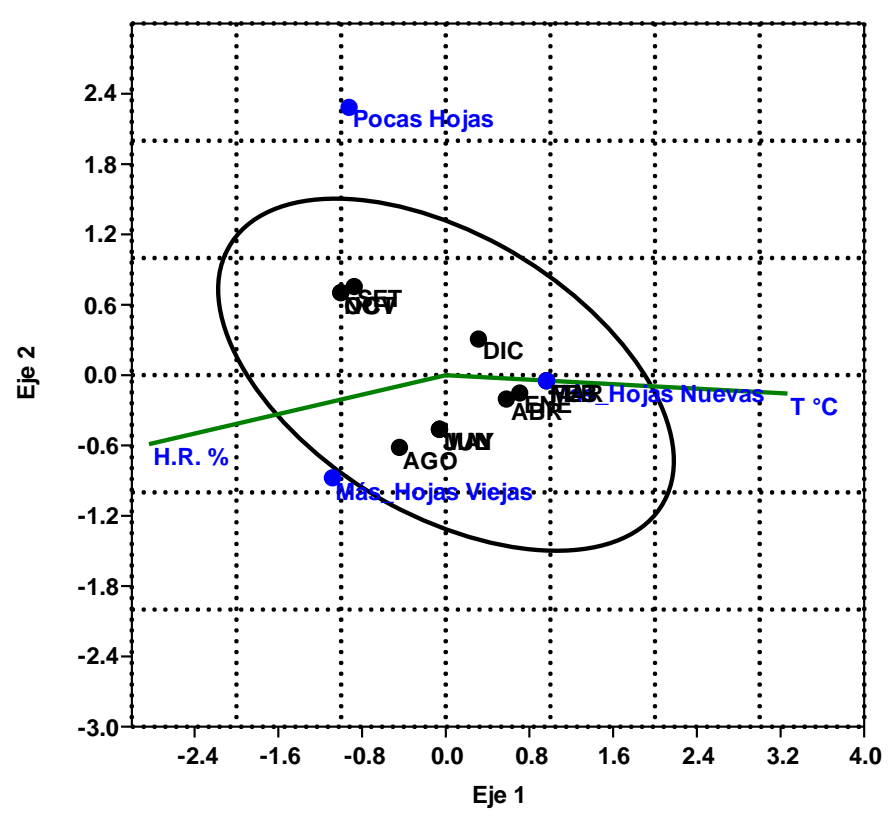

Figura 8. Análisis de Correlación Canónica de la Fenología Vegetativa de Caesalpinia pulcherrrima L. y los parámetros ambientales ( $\mathrm{T}{ }^{\circ} \mathrm{C}$ y H.R. \%) entre los meses de Enero 2006 y Diciembre 2008 .

respuesta (Figuras 1 y 2); esto explicaría porque siendo Enero el primer mes en el año que supera temperaturas de $24{ }^{\circ} \mathrm{C}$ esta especie no presentan botones florales ni floración alguna, empezando recién la fenofase de floración en el mes de Febrero. También esto explicaría por qué el pico máximo de floración se alcanza en el mes de abril, que es el mes siguiente a marzo en el que se produce la máxima temperatura del año. Una de las causas de demora de respuesta podría ser porque la producción de flores requiere de demandas altas de energía para formar tejidos no fotosintéticos y néctar (Ashman \& Schoen 1997), en un estudio realizado por Diekmann (1996) menciona que la suma de calor acumulado podría ser un buen predictor de floración. En relación al estadio Flor Marchita y Fruto Apareciendo se observan entre uno y dos meses de demora de respuesta con respecto a la temperatura. El estadio Fruto Verde presenta tres meses de demora de respuesta, mientras que el estadio Fruto Maduro muestra una demora de respuesta de hasta 6 meses. Al analizar las correlaciones de los estadios fenológicos con la humedad relativa media del aire (Figura 6), se observa que la humedad relativa está correlacionada positivamente con el estadio de Fruto Maduro, y muy poco relacionado con Fruto Verde, mientras que con la fenofase de floración y el estadio de Fruto Apareciendo muestra una correlación negativa. En la formación de hojas nuevas la temperatura juega también un papel importante, y en la formación de hojas viejas, la humedad es de significancia; a diferencia de las observaciones hechas en climas mediterráneos en los que el desarrollo de las hojas nuevas está principalmente controlado por la disponibilidad de humedad y después por la temperatura (DeBussche et al., 2004).

\section{Conclusiones.}

1.- $\quad$ La especie Caesalpinia pulcherrima (L.) Sw. presentó un claro patrón de floración anual entre los meses de febrero y agosto, teniendo pequeños estallidos de floración entre los meses de setiembre y de octubre.

2.- Las fenofases de fructificación mostraron diferencias entre ellas, por lo que no fue posible establecer un solo patrón de fructificación, la misma dificultad se obtuvo para establecer un solo patrón de foliación, para poder encontrarlos sería necesario realizar estudios de mayor tiempo de duración.

3.- La temperatura tiene un rol de gran significancia para la manifestación de las fenofases de floración, fructificación y foliación lo que concuerda con los estudios hechos por DeBussche et al., (2004).

4.- La humedad relativa mostró estar relacionada con la maduración de frutos y la maduración de hojas

5.- Hay demoras de respuesta de los estadios fenológicos en relación a la temperatura: un mes para la aparición de los estadios Botón Floral y Floración Total, dos meses para el estadio Flor Marchita, de tres a cuatro meses para el estadio Fruto Verde y de seis meses para el estadio Fruto Apareciendo.

\section{Agradecimientos.}

Al Dr. Edgar Sánchez por su orientación en los análisis de datos.

\section{Literatura citada.}

Alvarado M., Foroughbackhch R., Jurado E. \& Rocha A. 2002. El cambio climático y la fenología de las plantas. Ciencia UANL 5(4): 493 - 500

Ashman, T.L. \& Schoen, D.J.1997. The cost of floral longevity in Clarkia tembloriensis: an experimental investigation. Evolutionary Ecology, 11, 289-300.

Borges L.A., Sousa M. \& Lopes A.V. 2009. Phenology, pollination, and breeding system of the threatened tree Caesalpinia echinata Lam. (Fabaceae), and a review of studies on the reproductive biology in the genus. Flora 204: $111-130$

Brooke, M. de L., Jones, P.J., Vickery, J.A. \& Waldren, S. (1996) Seasonal patterns of leaf growth and loss, flowering and fruiting on a subtropical central Pacific island. Biotropica, 28: 164-179. 
Bullock S.H. \& Solis-Magallanes J.A.1990. Phenology of Canopy Trees of a Tropical Deciduous Forest in Mexico. Biotropica, 22 (1): 22-35 pp.

Cleland E.E., Chiariello N.R., Loarie S.R., Mooney H.A. \& Field C.B. 2006. Diverse responses of phenology to global changes in a grassland ecosystem. Proceedings of the National Academy of Sciences 103: 13740-13744.

Cruden R. W. \& Hermann-Parker S. M. 1979. Butterfly pollination of Caesalpinia pulcherrima, with observations on a psychophilous syndrome Journal of Ecology 67:155-168

DeBussche M., Garnier E. \& Thompson JD. 2004. Exploring the causes of variation in phenology and morphology in mediterranean geophytes: a genus-wide study of Cyclamen. Botanical Journal of the Linnean Society 145: 469-484.

Diekmann, M. 1996. Relationship between flowering phenology of perennial herbs and meteorological data in deciduous forests of Sweden. Canadian Journal of Botany, 74: 528-537.

Fenner, M.1998. The phenology of growth and reproduction plants. Perspectives in Plant Ecology, Evolution and Systematics 1: 78-91 pp.

Fuentes V., Granda M.M., Lemes Hernández C. M. \& Rodríguez Ferrada C. 2000. Estudios Fenológicos en Plantas Medicinales XI. Rev. Cubana Plant. Med. 5:106-113.

Govindarajan M., Mathivanan T., Elumalai K., Krishnappa K. \& Anandan A. 2011. Mosquito larvicidal, ovicidal, and repellent properties of botanical extracts against Anopheles stephensi, Aedes aegypti, and Culex quinquefasciatus (Diptera: Culicidae). Parasitol Res. 109(2):353-67.

Gregg J.W., Jones C.G. \& Dawson T.E. 2003. Urbanization effects on tree growth in the vicinity of New York City. Nature 424: 183-187.

Harper G.H., Mann D.G., Thompson R. 2004. Phenological monitoring at Royal Botanic Garden Edinburgh. Sibbaldia 2: 33-45.

HOLDRIDGE, L. 2000. Ecología Basada en Zonas de Vida. Instituto Interamericano de Cooperación para la Agricultura (HCA). Quinta Reimpresión. San José Costa Rica.

Jordano P. 1992. Fruits and frugivory. Seeds In: Fenner M, (eds). The Ecology of Regeneration in Plant Communities. CAB International, Oxford. pp. 105-156.

Lorenzi H. \& Souza H. M. 2008. Plantas ornamentais no Brasil: arbustivas, herbáceas e trepadeiras. 4. ed. Nova Odessa: Instituto Plantarum.

Loubry D. 1994. La phénologie des arbres caducifoliés en forêt guyanaise (5 de latitude nord): Illustration d'un déterminisme à composantes endogène et exogène. Canadian Journal of Botany 72:1843-1857.

Mejía K. \& Rengifo E. 1995. Plantas Medicinales de Uso popular en la Amazonia Peruana. Agencia Española de Cooperación Internacional. Lima. 2da edición. 286 pp.

Newstrom L.E., Frankie G.W. \& H. G. Baker. 1994. A new classification for plant phenology base on flowering patterns in lowland tropical rain forest trees at La Selva, Costa Rica. Biotropica:26 (2): 141-159
N.E.R.A.G. 2001. New England regional assessment. Durham, NH, USA: University of New Hampshire, Institute for the Study of Earth, Oceans, and Space.

Paguagua D. \& Soto A. 2010. Arbores y Arbustos de la Ciudad de León. Instituto Nacional de Biodiversidad. Editorial INBio. Costa Rica. 133 pp.

Pinheiro A.L. \& Almeida E.C. 2000. Fundamentos de taxonomía e dendrologia tropical: metodología dendrológica.v2. Vicosa: SIF. 188pp.

Primack R.B. \& Miller-Rushing A.J. 2009. The role of botanical gardens in climate change research. New Phytologist 182: 303-313.

Pullaiah T. 2006. Encyclopedia of World Medicinal Plants, Vol 1. New Delhi: Regency publications 1680 pp.

Rojas F. \& Torres G. 2009. Árboles del Valle Central de Costa Rica: reproducción. Hoja Sen. Kurú: Revista Forestal (Costa Rica): 6(17).

Rondón J. 1994. Sinopsis de las principales metodologías aplicadas a los estudios fenológicos de los árboles tropicales. Revista Forestal Latinoamericana 14:521531.

Rosenzweig C., Karoly D., Vicarelli M., Neofotis P., Wu O., Casassa G. Menzel A., Root T.L., Estrella N., Seguin B., Tryjanowski P., Liu C., Rawlins S. \& Imeson A. 2008. Attributing physical and biological impacts to anthropogenic climate change. Nature 453:353-357.

Rusch, V.E.1993. Altitude variation in the phenology of Nothofagus pumilio in Argentina. Revista Chilena de Historia Natural 66:131-141.

Selvam. 2007. Trees and shrubs of the Maldives. RAP Publication No. 2007/12 Thammada Press Co.,Ltd., Bangkok. 246pp

S.E.C.F. 2005. Diccionario forestal. Publicado por MundiPrensa Libros, Madrid. 1314 pp.

Soto A. 2010. Plantas con flores que atraen mariposas. 1ed Santo Domingo de Heredia, Costa Rica. Instituto Nacional de Biodiversidad INBio. 144 p.

Sudhakar M., Rao Ch. B., Rao P.M., Raju D.B. \& Venkateswarlu Y. 2006. Antimicrobial activity of Caesalpinia pulcherrima, Euphorbia hirta and Asystasia gangeticum. Fitoterapia Vol 77(5): 378-380.

Takawale H., Mute V., Awari D., Hukkeri V.I., Mehta P. \& Vawhal P. 2011. Screening of Antiulcer Activity of Caesalpinia pulcherrima L. Bark. against Aspirin Induced Ulcer in Rats. World Journal of Medical Sciences 6 (4): 168-172.

Williams, R.J., Myers, B.A., Muller, W.J., Duff, G.A. \& Eamus, D. (1997) Leaf phenology of Woody species in a North Australian tropical savanna. Ecology 78: 25422558.

Wright, S.J. \& Van Schaik, C.P. 1994. Light and the phenology of tropical trees. American Naturalist, 43 192-199.

Zepeda C. \& Velazquez E. 1999. El bosque tropical caducifolio de la vertiente sur de la sierra de Nanchititla, Estado de Mexico: la composicion y la afinidad geografica de su flora. Acta Botánica Mexicana 46: 2955. 
Ecol. apl. Vol. 14 № 2, pp. 201-209

${ }^{1}$ rcastro@lamolina.edu.pe. Jardín Botánico “Octavio Velarde Núñez”. Departamento Académico de Biología, Universidad Nacional Agraria La Molina. Av. La Molina s/n, Lima 12, Perú. Apartado postal: 12-056, Perú.

2 vcastro@lamolina.edu.pe. Jardín Botánico “Octavio Velarde Núñez”. Departamento Académico de Biología, Universidad Nacional Agraria La Molina. Av. La Molina s/n, Lima 12, Perú. Apartado postal: 12-056, Perú.

${ }^{3}$ aceroni@lamolina.edu.pe. Jardín Botánico “Octavio Velarde Núñez”. Departamento Académico de Biología, Universidad Nacional Agraria La Molina. Av. La Molina s/n, Lima 12, Perú. Apartado postal: 12-056, Perú. 